\title{
Unusual causes of upper gastrointestinal bleeding: Review of Chinese literature
}

\author{
Wei Zhang ${ }^{1}$, Eliakim Manda ${ }^{1,2}$, Ming Qiu ${ }^{1, *}$ \\ ${ }^{1}$ Department of General Surgery, Changzheng Hospital Affiliated to Second Military Medical Hospital, Shanghai, China; \\ ${ }^{2}$ Department of General Surgery, Zambia Army Hospital, Lusaka, Zambia.
}

\begin{abstract}
Summary Upper gastrointestinal bleeding (UGB) is a life-threatening complication of gastrointestinal diseases. There is a large variety of uncommon reasons which contribute to UGB and might become reasons for misdiagnosis and sometimes lead to fatal consequences. In this review, clinical characteristics of uncommon causes of UGB reported in the Chinese literature are summarized.
\end{abstract}

Keywords: Upper gastrointestinal bleeding, unusual cause, China

\section{Introduction}

Upper gastrointestinal bleeding (UGB) is a lifethreatening complication of gastrointestinal diseases. Even though bleeding is the common symptom, different causes of gastrointestinal bleeding are significantly different. Not only the gastrointestinal tract itself, but also lesions in adjacent organs and systemic diseases contribute to bleeding. Therefore, realization of the etiology and characteristics is essential for doctors to diagnose and treat the disease (1).

Peptic ulcer remains the most common cause of UGB, representing approximately half of all cases $(2,3)$. Esophageal varicose and gastritis $(4,5)$, gastric carcinoma (5) and benign tumors of the digestive tract (6) are also common causes of UGB. There are many case reports of unusual causes of UGB (7-9), hence widening our understanding into the etiology of UGB. On the other hand, UGB of rare diseases can also be the reason for misdiagnosis (10) and sometimes leads to fatal consequences (11).

In this short review, we try to summarize rare causes of UGB from a Chinese doctors' point of view. This

\footnotetext{
*Address correspondence to:

Dr. Ming Qiu, Department of General Surgery, Changzheng Hospital Affiliated to Second Military Medical University, No.415 Fengyang Road, Shanghai 200003, China.

E-mail: qiuming2006@yahoo.cn
}

will enrich our knowledge to the etiology of UGB and broaden our judgment in the process of diagnosis of UGB, and thus improve the accuracy of diagnosis and efficacy of treatment.

\section{Search strategy and data extraction}

The electronic databases of Chinese Medicine, Chongqing VIP, from January 1989 to June 2011 were searched to identify all clinical reports related to UGB. The disease-specific search term UGB was used. The abstracts were reviewed and only those reported rare causes of UGB (that is, those other than peptic ulcer, gastric carcinoma, gastritis, varicose rupture and gastric polyps) were included. The full text was read and clinical related data was extracted, summarized and listed as follows.

\section{Unusual causes of UGB}

\subsection{Crest syndrome}

Crest syndrome is a form of systemic scleroderma associated with antibodies against centromeres that usually spares the kidneys. It is extremely uncommon for Crest syndrome to present with UGB and can be easily misdiagnosed with hemorrhage following variceal rupture. The following features are helpful for the establishment of diagnosis: i) Crest syndrome is a connective tissue disease and multiple organs are involved, ii) rupture of capillaries is the basic pathologic change and both the upper and lower gastrointestinal 
tract can be the source of bleeding, iii) liver damage is slight and coagulation function is normal, thus the bleeding can stop spontaneously and $i v$ ) laboratory tests show that anticardiolipin antibody is positive, which is the characteristic finding of Crest syndrome (12).

\subsection{Dieulafoy's lesion}

Dieulafoy's lesion is a submucosal caliber-persistent artery anomaly. The exact frequency of Dieulafoy's lesion is difficult to estimate. The patients usually have no history of peptic ulcer and liver cirrhosis but the clinical presentation mimics these situations because blood loss is usually significant and rapid. Rahbour et al. (13) reviewed the English literature and found that $95 \%$ of Dieulafoy's lesion occurs in the upper part of the stomach, within $6 \mathrm{~cm}$ of the gastroesophageal junction and commonly in the lesser curvature. However, this trend seemed to be not so obvious in the Chinese literature as there are still many cases with a lesion located in the antrum or even the duodenum. Another characteristic feature of Dieulaoy's lesion we summarized after reviewing the Chinese literature is periodic attacks. The bleeding stops temporarily with hypovolemic shock but recurs while resuscitation is achieved. The key point of successful treatment lies in the location of the lesion. But the extent of the lesion is limited and can be missed by endoscopy, and thus sometimes leads to fatal consequences $(14,15)$.

\subsection{Mallory-Weiss syndrome}

Mallory-Weiss syndrome refers to a tear or laceration of the mucous membrane, most commonly at the point where the esophagus and the stomach meet (gastroesophageal junction). In the Chinese literature, more than half of the patients had a history of drinking which caused nausea and vomiting. The rapid increase of intra-gastric or intra-abdominal pressure accounted for the tear of mucosa at the gastroesophageal junction which is less flexible. The typical symptom is that vomiting of gastric contents followed by hematemesis, which typically begins with vomiting fresh bright red blood which becomes dark gradually. The diagnosis can be established according to clinical presentation and be confirmed by endoscopic examination (16). Atkinson (17) believed that the presence of a small hiatal hernia appeared to predispose to mucosal tears. However, there are no reports describing this condition in the Chinese literature.

\subsection{Rupture of aneurysm}

UGB originating from rupture of an aneurysm of the branches of the celiac artery or superior mesenteric artery is rare but the mortality rate could be higher than $75 \%$. Chen et al. (18) summarized that these patients usually had no specific symptoms before the hemorrhage attack but the following features were valuable for the establishment of diagnosis: i) blood loss was rapid and massive and the patients quickly entered a state of shock, ii) a pulsatile mass could be found on physical examination and iii) endoscopy could not find the source of bleeding but angiography was the most valuable means to locate it.

\subsection{Aortoenteric fistula}

Aortoenteric fistula, a rare cause of gastrointestinal bleeding, is defined as a communication between the aorta and the gastrointestinal tract. It can be divided into: i) primary aortoenteric fistula with no history of aorta surgery but originates from erosion into the gastrointestinal tract (usually the fourth part of duodenum) with expansion of the aneurysm and ii) secondary aortoenteric fistula, which is mostly a complication of surgery, such as rupture of an anastomosis in the aorta, or the erosion of the graft into the duodenum or small intestine. The classic triads of symptoms include abdominal pain, gastrointestinal bleeding and pulsating abdominal mass. Jin et al. summarized that more than $90 \%$ Chinese patients had slight hematochezia before massive bleeding which could be considered as warning signs of massive hemorrhage (19). Early surgical intervention was the only cure for this condition $(19,20)$. Apparently, the diagnosis is relatively easy in secondary aortoenteric fistula as the patients have a clear history of aorta surgery. However, because aorta surgery has developed very late in China, the majority of aortoenteric fistulas are primary and not secondary and even a history of an aorta aneurysm can hardly be a question (19). This adds to the difficulty of establishing a prompt diagnosis.

\subsection{Ancylostomiasis}

Ancylostomiasis (also anchylostomiasis or ankylostomiasis) is the condition of infestation by ancylostoma hookworms. It has become a less serious public health problem in Western countries but a report showed that the average infestation rate of ancylostoma hookworms was $17.2 \%$ in 2000 in China. Jing (21) reported 40 cases of ancylostomiasis-related UGB and found that all the patients lived in a rural area and/or presented with hematemesis or hematechezia and chronic anemia. More than $75 \%$ of the patients suffered discomfort in the upper abdomen and more than $65 \%$ presented with fatigue and bad appetite. Some patients had strange food addictions and hookworm dermatitis. The diagnosis could be established according to the positive result of hookworm ovum and the direct finding of hookworm in the stomach. 


\subsection{Small intestine ulcer}

Gao (22) reported a case of small intestine ulcer induced by adhesion. In laparotomy, after exclusion of other sources of hemorrhage, a $20 \mathrm{~cm}$ long jejunum was found tightly adherent to each other and could not be lysed. Fresh blood accumulated in the lumen of the intestine. After resection of this part of the small intestine, $6 \mathrm{~cm} \times 4 \mathrm{~cm}$ ulcers were found on either side of the adhesion. Local ischemia induced by chronic obstruction and long-term distension might have been responsible for the formation of the ulcers.

\subsection{Crohn's disease}

Crohn's disease can be a cause of intestinal bleeding or obstruction. But it is a rare disease in China and an even rarer condition combining bleeding and obstruction if a patient suffers from Crohn's disease. If the surgeon is satisfied with the resolution of obstruction during the first laparotomy, a second operation might become necessary because of post-operative bleeding. Wu et al. (23) reported a case of UGB whose pre-operative $\mathrm{X}$-ray film also showed signs of ileus. During the operation, after lysis of all adhesions, a short segment of jejunum of $10 \mathrm{~cm}$ from Tretiz's ligament was found to be edematous. The pathological examination of this resected intestine showed the typical changes of Crohn's disease with fresh blood inside the lumen. The author believed that UBG was not only the consequence of ileus, but also the co-existing condition. So, resection of the lesion might be a better choice than short-circuit surgery.

\subsection{Ascariasis}

Ascariasis is a very common condition in children. Sangkhathat et al. (24) reported two cases of ascariasisrelated UGB in English. In one case, a roundworm was found adhering to an oozing duodenal ulcer and in another case, numerous ascaris lumbricoides lead to a segmental volvulus of the distal ileum, to gangrene and perforation. Huang et al. (25) reported another case in which a mass of tapeworms was found piled up in the lumen and mechanical pressure induced mucosal erosion and was the source of bleeding.

\subsection{Rabies}

Wang et al. (26) reported a case of rabies combined with UGB. The patient was admitted with a history of hematemesis. Conservative therapy was not effective and further inquiry found a history of a dog bite 25 days prior to presentation. The patient presented with typical symptoms of rabies and eventually died of hypovolemic shock and lung dysfunction. The bleeding might have been a type of stress ulcer. Hyperfunction of the sympathetic nerves from the invasion of virus into the brain tissue might be the underlying pathogenesis. 3.11. Benign tumor of the duodenum

Benign tumors of the duodenum, such as leiomyoma (27), Brunner adenoma (28), lymphoceles (29) and hemangioma (30) are rare conditions. The formation of an ulcer with the growth of tumor can be the source of bleeding. In most circumstances, the hemorrhage is mild. Patients with large tumors present with symptoms of bleeding combined with intestinal obstruction. Endoscopy and a barium meal are the most valuable means to locate the lesion while ultrasonography is not feasible because of interference from intestinal gas. Pre-operative diagnosis is usually difficult because of the difficulty of obtaining a specimen for pathological examination. It can only be established following surgical resection.

\subsection{Rare conditions of the stomach}

There are reports of UGB related to lymphoepithelioma (31), lymphoma of mucosa associated lymphoid tissue (32), fibrolipoma (33) and inflammatory myofibroblastic tumor (34) of the stomach in the Chinese literature. The common feature of these uncommon causes of UGB is that the diagnosis can hardly be established before surgery. Fortunately, UGB with an obvious lesion in the stomach itself is a definite indication for surgical intervention and the diagnosis can only be confirmed by pathological examination with the assistance of immunohistochemistry detection of a specific antigen for a particular condition.

\subsection{Pancreas disorders}

Solid pseudopapillary tumor of the pancreas (SPTP), an uncommon neoplasm with low malignant potential often causes few symptoms and may reach a large size before it is detected. It can invade adjacent structures, mostly the duodenum, and cause ulcers and hemorrhage (35). Pseudo-cyst of the pancreas shares some common features with SPTP despite the fact that the patients have a clear history of pancreatitis (36). Both lesions can be easily located on ultrasonography or CT scans and the final diagnosis needs pathological confirmation. Zhu et al. (37) summarized their experience of treating pancreatogenic portal hypertension, rupture of varices of gastric fundus complicated with hemorrhage of the splenic artery, pancreatic duct, or pancreatic pseudocyst fistula. They concluded that the characteristic manifestations of the disease were history of chronic pancreatitis, long-term intermittent pain in the epigastric region followed by melena, vomiting of blood, and splenomegaly with normal hepatic function. The key steps of diagnosing the disease were gastroduodenal endoscopy, retrograde pancreatocholangiography and 
selective splenic arteriography. Li et al. (38) reported a case of UGB whose endoscopic examination showed an ulcer in the duodenum. CT scan indicated a mass on the head of the pancreas and the final pathological diagnosis was malignant insulinoma. The author concluded that some neuroendocrine tumors of the pancreas can lead to UGB via two approaches: i) direct invasion and ii) influence of hormone levels leading to ulcer formation.

\subsection{Behcet's disease}

Behcet's disease symptoms vary from person to person. Most symptoms of the disease are caused by vasculitis. Inflammation and ulceration throughout the digestive tract caused by Behcet's disease is a less common symptom. Wei et al. (39) reported a case of UGB with Behcet's disease. Under endoscopy, multiple ulcers in the esophagus and cardia were detected. The patient had a clear history of recurrent mouth and genital sores, a characteristic skin lesion on the face, and the diagnosis of Behcet's disease with UGB was established.

\section{Comment}

UGB is a common emergency situation needing prompt and accurate diagnosis. Even though the common causes of UGB, such as peptic ulcer and variceal bleeding, account for more than 80 percent of cases, there is still a large variety of uncommon reasons which contribute to UGB with digestive tract bleeding being only part of the symptoms in a certain case.

The spectrum of diseases is different between Western countries and China. We should mention that some rare causes contributing to UGB might be very common in Western countries, for example, Crohn's disease. There were still some really rare causes, for example, UGB derived from small intestine ulcer and rabies, which had not been reported in the English literature. Despite that, what we emphasized in this mini-review was the clinical features of these rare conditions, especially their differences from those reported in the English literature. We hoped to provide evidence that might not be easily accessed by nonChinese physicians and share our experience with foreign doctors.

A doctor should bear in mind that some rare diseases can cause rapid and massive hemorrhage and that the mortality rate could be higher than 75 percent. That means that not all cases ameliorate with conservative treatment and prompt surgical intervention is the only cure under these circumstances.

Another viewpoint is that not all sources of bleeding can be located by endoscopy. So the rational combination of endoscopy with other imaging detection, such as ultrasonography, CT scan and angiography offers more valuable information for achievement of diagnosis.

There are still some clues for diagnosis of uncommon cases. The doctor's concentration or focus should not only be limited to the digestive tract itself. A detailed history inquiry and thorough physical examination may lead the doctor to the correct diagnosis as even the rare diseases have their own characteristics.

\section{References}

1. Suo J. Causes and features of gastrointestinal bleeding. Chinese Journal of Practical Surgery. 2010; 30:422-425. (in Chinese)

2. Bor S, Dağli U, Sarer B, Gürel S, Tözün N, Sıvrı B, Akbaş T, Sahın B, Memık F, Batur Y. A retrospective study demonstrating properties of nonvariceal upper gastrointestinal bleeding in Turkey. Turk J Gastroenterol. 2011; 22:249-254.

3. Huang PN. Causes of acute upper gastrointestinal bleeding. Chinese Journal of Gerontology. 2010; 30:2846-2847. (in Chinese)

4. Hearnshaw SA, Logan RF, Lowe D, Travis SP, Murphy MF, Palmer KR. Acute upper gastrointestinal bleeding in the UK: Patient characteristics, diagnoses and outcomes in the 2007 UK audit. Gut. 2011; 60:1327-1335.

5. Chen LH, Wan ZQ. Causes and risk factors analysis of upper gastrointestinal bleeding. Clin Focus. 2003; 18:200-201. (in Chinese)

6. Bassi DG, Silva FP, Sousa FM. GIST induced upper gastrointestinal bleeding. Rev Assoc Med Bras. 2010; 56:520-521.

7. Mantoo SK, Rao J. An unusual cause of upper gastrointestinal bleeding. Endoscopy. 2010; 42 (Suppl 2): E123.

8. Chen KC, Yen HH, Chen YY. Unusual cause of upper gastrointestinal bleeding. Gastroenterology. 2011; 141: e11-12.

9. Chen CJ, Hsu HT, Yen HH. An unusual cause of upper gastrointestinal bleeding. Gastric yolk sac tumor with a large retroperitoneal metastasis. Gastroenterology. 2010; 139:1098, 1427.

10. Li FH. 3 cases of misdiagnosis of upper gastrointestinal bleeding. Chinese Journal of Misdiagnostics. 2011; 11:2909. (in Chinese)

11. Fu Q, Chen G. A case of fatal gastrointestinal bleeding with uncommon cause. Journal of Tianjin Medical University. 1997; 3:70-71. (in Chinese)

12. Wu Q, He XD, Tao LY, Cai L. Diagnosis and treatment of CREST syndrome combined with upper gastrointestinal bleeding. Chinese Journal of Digestive Surgery. 2011; 10:139-140. (in Chinese)

13. Rahbour G, Ullah MR, Siddiqui M, Agha R, Kerwat R. Dieulafoy lesion endoscopically rubber banded, with further severe haematemesis requiring emergency laparotomy - Case report. Int J Surg Case Rep. 2011; 2:154-156.

14. Zhang XY, Liu NQ, Lv PF. Dieulofy's lesion with upper gastrointestinal bleeding: 13 cases reports. Chinese Journal of Postgraduates of Medicine. 2005; 28:31-33. (in Chinese)

15. Jing N, Cai JT. Proceedings in treatment of Dieulafoy lesion. Zhejiang Medical Journal. 2007; 29: 296-298. (in 
Chinese)

16. Shen XD. Treatment of Mallory-Weiss syndrome (30 cases experience). Chinese Community Doctors. 2011; 13:40. (in Chinese)

17. Atkinson M, Botterill MB, Edwards AT, Mitchell WM, Peet BG, Williams RE. Mucosal tears at the oesophagogastric junction (the Mallory-Weiss syndrome). Gut. 1961; 2:1-11.

18. Chen FZ, Wu ZQ, Xu WS. Upper gastointestinal bleeding from the rupture of aneurysm (23 cases report). Chinese Journal of Practical Surgery. 1994; 14:400-402. (in Chinese)

19. Jin XH, Chen CX. Clinical analysis of 21 cases of aortoenteric fistula in China. Zhejiang Medical Journal. 2010; 32:737-738. (in Chinese)

20. Ma F, Yu B. A case report of gastrointestinal bleeding from aorto-duodenum fistua. Chinese Journal of Difficult and Complicated Cases. 2010; 9:574. (in Chinese)

21. Jiang Y. 40 cases of gastrointestinal bleeding with ancylostomiasis. Hebei Medical Journal. 2010; 16:79-81. (in Chinese)

22. Gao CX. Gastrointestinal bleeding from rare causes: Eight cases reports. Jiangsu Medical Journal. 1999; 25:145. (in Chinese)

23. Wu SS, Zeng QL. Experience of treatment of upper gastrointestinal bleeding with uncommon causes. Jiangxi Medical Journal. 1994; 29:340-341. (in Chinese)

24. Sangkhathat S, Patrapinyokul S, Wudhisuthimethawee $\mathrm{P}$, Chedphaopan J, Mitamun W. Massive gastrointestinal bleeding in infants with ascariasis. J Pediatr Surg. 2003; 38:1696-1698.

25. Huang ZN, Lan JG. Gastrointestinal bleeding induced by ascariasis: A case report. Chinese Journal of Clinical Gastroenterology. 2002; 14:40. (in Chinese)

26. Wang YX, Tian X. A case of gastrointestinal bleeding with rabies. Chinese Community Doctors. 2004; 6:43-44. (in Chinese)

27. Zhang YY, Zhang YJ, Huo L, Han BJ, Liu Y. Treatment of gastrointestinal bleeding induced by duodenal leimyoma, 3 cases reports. Chinese Journal of Practical Surgery. 2007; 24:437. (in Chinese)

28. Liu XL, Zong PJ, Shi JS, ZhongYM, Feng X. A case of duodenal Brunner adenoma. Chinese Journal of Gastrointestinal Surgery. 2008; 11:127. (in Chinese)

29. Ma SG, Wu JS, Wu M. A case of lymphoceles in the second part of duodenum. Chinese Journal of Digestive Endoscopy. 2008; 25:498. (in Chinese)

30. Liu BQ, Liu YH. A case of duodenum hemangioma with ulcer. Chinese Journal of Practice Medicine. 2003; 19:599. (in Chinese)

31. Lou SX, Shi HQ, Zhai XL. A case of lymphoepithelioma. Chinese Journal of Pathology. 2005; 34:759-760. (in Chinese)

32. Li B. A case of gastric lymphoma of mucosa associated lymphoid tissue combined with gastrointestinal bleeding. China Medicine. 2010; 5:62. (in Chinese)

33. Meng XY, Xie XJ, Wang Q. A case of gastric fibrolipoma combined with gastrointestinal bleeding. Chinese Journal of Digestive Endoscopy. 2010; 27:426. (in Chinese)

34. Li CX, Liu YQ, Liu JX, Bai XQ, Liu WY. Gastric inflammatory myofibroblastic tumor. Chinese Journal of Digestion. 2010; 30:857. (in Chinese)

35. Cui YF, Wang GH, Tai S, Zhong XY. A case of gastrointestinal bleeding induced by SPTP. Chinese Journal of Current Advances in General Surgery. 2009; 12:180-182. (in Chinese)

36. Zhang FG, Wu TJ, Sun HD. A case of gastrointestinal bleeding from internal fistula from the pseudo-cyst of pancreas and the stomach. Journal of Community Medicine. 2011; 9:39. (in Chinese)

37. Zhu W. Pancreatogenic portal hypertension associated with hemorrhage of pancreatic duct or upper digestive tract. Report of 2 cases. Journal of Abdominal Surgery. 2002; 15:335-336. (in Chinese)

38. Li M, Fang MZ. A case of malignent insulinoma. Chinese Journal of Misdiagnostics. 2009; 9:6787. (in Chinese)

39. Wei XQ, Zhang M. A case of esophageal Behcet's disease. Chinese Journal of Digestion. 2005; 25:699. (in Chinese)

(Received January 12, 2012; Revised January 21, 2012; Accepted January 31, 2012) 